\title{
An Improved Tunnel-Track Model in Saturated Poroelastic Soils to a Moving Point Load
}

\author{
Zunan Fu, ${ }^{1}$ Yuanlei Xu $\mathbb{D}^{2},{ }^{2}$ Zonghao Yuan $\left(\mathbb{D},{ }^{3}\right.$ Li Shi $\left(\mathbb{D},{ }^{3}\right.$ Guoshuai Wang, ${ }^{4}$ Wenbo Song, ${ }^{4}$ \\ and Yanming $\mathbf{Y u} \mathbb{D}^{1}$ \\ ${ }^{1}$ Huadong Engineering Corporation Limited, Hangzhou 311122, China \\ ${ }^{2}$ Zhejiang Academy of Building Research \& Design Co., Ltd, Hangzhou 310023, China \\ ${ }^{3}$ Zhejiang University of Technology, Hangzhou 310023, China \\ ${ }^{4}$ Zhejiang Huadong Engineering Construction Management Co., Ltd, Hangzhou 311122, China
}

Correspondence should be addressed to Yuanlei Xu; zjj.xuyuanlei@zjsjky.com and Zonghao Yuan; yuanzh@zjut.edu.cn

Received 2 August 2021; Accepted 29 October 2021; Published 24 November 2021

Academic Editor: Meng Gao

Copyright (C) 2021 Zunan Fu et al. This is an open access article distributed under the Creative Commons Attribution License, which permits unrestricted use, distribution, and reproduction in any medium, provided the original work is properly cited.

To predict the mechanical response of a circular cavity/tunnel buried in saturated poroelastic soils to a moving point load, a semianalytical model is provided in this work. The soils are governed by Biot's theory that describes the wave propagations for saturated poroelastic materials. The displacement and stress vectors for the solid skeleton and pore-water fluid are represented by scalar and vectorial potentials. The governing equations for the tunnel and surrounding soils are solved in the frequency domain with the aid of separation of variables and Fourier transformations. To check the feasibility of the present analytical model, the solution is compared with other available results calculated for the ring load case. The good agreement shows the correctness of the present model. Numerical results suggest that the mechanical response from a moving point load in a tunnel for two-phase poroelastic materials is quite different from that in single-phase elastic materials. The critical velocity of the tunnel-soil system is around the shear wave speed of soils while the second one introduced into the track-tunnel-soil system with very high value is around the critical velocity of the track structure itself.

\section{Introduction}

Recently, the propagations of ground vibration from underground railways into nearby buildings have become an important research topic due to the fact that more and more metro lines run in urban areas and become closer to the nearby buildings. Lots of research works have been done to investigate this problem through numerical simulations, such as FEM, BEM, and coupled FEM-BEM [1-3]. The analytical models can predict the vibrations from underground in a relatively faster way compared with numerical simulations. The reasons may lie in the fact that the analytical solution does not need the element discretization or the addition of energy attenuation boundaries to the discretized domain. Since numerical simulations are quite popular, in the present work, only some representative analytical models are discussed.
As mentioned above, some typical research works on the topic of analytical modeling on underground railway-induced vibrations are introduced briefly in the following. Metrikine and Vrouwenvelder [4] inserted an Euler beam into the ground to model the tunnel-soil system while the moving train is simplified as a moving point load running on the beam. Krylov [5] regarded each sleeper in the underground tunnel as a point vibration source buried in the halfspace, and with the help of Green functions, the response at arbitrary positions can be calculated. However, this buried source model is only suitable for predicting vibrations due to low-frequency propagated waves. Forrest and Hunt [6] proposed an analytical model for the vibration calculation around the tunnel, which can be used to predict the ground vibration at the vibration source. To model the twin tunnel case, Guan and Moore [7] gave an analytical solution for computing the mechanical response from two cylindrical 
cavities buried in an unbounded space and investigated the interaction problem between two deep side-by-side circular tunnels.

All above studies deal with a tunnel embedded in singlephase elastic soils; however, there exists underground water in soils meaning that the soils are two-phase material instead of single phase. The presence of pore water in soils may have significant effects on wave propagations generated by the underground moving trains. Therefore, it may be safe to argue that the fully saturated poroelastic soil model is superior to the elastic one for the analysis of mechanical responses of the metro system or at least the poroelastic soil models are as good as the elastic soil one.

Regarding the two-phase poroelastic soil model, Senjuntichai and Rajapakse [8] modeled the transient response of poroelastic full space with a cylindrical cavity under symmetric axial loadings. Using a similar model, Lu and Jeng [9] investigated the dynamic response from a cavity embedded in poroelastic soils. Based on the work by Forrest and Hunt [6] and Lu [9], in this paper, an improved tunneltrack model in saturated poroelastic soils to a moving point load is proposed by semianalytical methods. The soil is governed by Biot's theory which describes the wave transmissions for saturated poroelastic materials. The displacement and stress vectors for the solid skeleton and pore-water fluid are represented by scalar and vectorial potentials. To check the correctness of the present analytical model, the solution is compared with other results calculated for the ring load case. Using the boundary conditions along the tunnel surface and the full space, the formulas written for the displacement, stress, and pore pressure are provided.

\section{Governing Equations and General Solutions}

The governing equations of motion of fully saturated poroelastic soils are as follows:

$$
\begin{aligned}
& \mu \mathbf{u}_{i, j j}+\left(\lambda_{c}+\mu\right) \mathbf{u}_{j, j i}+\alpha M \mathbf{w}_{j, j i}=\rho \ddot{\mathbf{u}}_{i}+\rho_{f} \ddot{\mathbf{w}}_{i}, \\
& \alpha M \mathbf{u}_{j, j i}+M w_{j, j i}=\rho_{f} \ddot{\mathbf{u}}_{i}+m \ddot{\mathbf{w}}_{i}+b \dot{\mathbf{w}}_{i},
\end{aligned}
$$

where $\mathbf{u}_{i}$ and $\mathbf{w}_{i}$ are solid and fluid displacement vectors; $i$ denotes the directions $x, y$, and $z$; the subscripts ${ }_{i, j j}$ and ${ }_{j, j i}$ represent the derivatives of variable component; the dot is the differential over time; $\lambda_{c}=\lambda+\alpha^{2} M$ and $\mu$ are equivalent Lame constants for poroelastic soils; $\rho$ and $\rho_{\mathrm{f}}$ are the mixture density of two-phase soils and water; $m=\rho_{\mathrm{f}} / n$ with $n$ as the porosity; and $b$ is the ratio between the water viscosity and permeability of the medium. The constitutive equation is as follows:

$$
\begin{aligned}
\sigma_{i j} & =\lambda \delta_{i j} \theta+\mu\left(\mathbf{u}_{i, j}+\mathbf{u}_{j, i}\right)-\alpha \delta_{i j} p, \\
p & =-\alpha M \theta+M \varsigma, \\
\varsigma & =-\mathbf{w}_{i, i},
\end{aligned}
$$

where $\theta=\mathbf{u}_{i, i}$ is solid strain; $\sigma_{i j}$ is stress tensor; and $p$ is the pore-water pressure.

The displacement vectors of solid and fluid phases are expressed by the scalar and vector potentials [6]:

$$
\begin{aligned}
& \mathbf{u}=\nabla \varphi+\nabla \times \boldsymbol{\psi}, \\
& \mathbf{w}=\nabla \chi+\nabla \times \boldsymbol{\Theta},
\end{aligned}
$$

where $\varphi$ and $\psi$ are scalar and vector potentials of solid phase while $\chi$ and $\Theta$ are those of pore fluid. Substituting equations (4) and (5) into equations (1) and (2), the following equations are given:

$$
\begin{gathered}
{\left[\begin{array}{cc}
\lambda_{c}+2 \mu & \alpha M \\
\alpha M & M
\end{array}\right]\left[\begin{array}{c}
\nabla^{2} \varphi \\
\nabla^{2} \chi
\end{array}\right]=\left[\begin{array}{cc}
\rho \frac{\partial^{2}}{\partial t^{2}} & \rho_{f} \frac{\partial^{2}}{\partial t^{2}} \\
\rho_{f} \frac{\partial^{2}}{\partial t^{2}} & m \frac{\partial^{2}}{\partial t^{2}}+i \frac{\partial}{\partial t} b
\end{array}\right]\left[\begin{array}{l}
\varphi \\
\chi
\end{array}\right]} \\
{\left[\begin{array}{ll}
\mu & 0 \\
0 & \mu
\end{array}\right]\left[\begin{array}{c}
\nabla^{2} \psi \\
\nabla^{2} \Theta
\end{array}\right]=\left[\begin{array}{cc}
\rho \frac{\partial^{2}}{\partial t^{2}} & \rho_{f} \frac{\partial^{2}}{\partial t^{2}} \\
\rho_{f} \frac{\partial^{2}}{\partial t^{2}} & m \frac{\partial^{2}}{\partial t^{2}}+\frac{\partial}{\partial t} b
\end{array}\right]\left[\begin{array}{l}
\psi \\
\Theta
\end{array}\right] .}
\end{gathered}
$$

Transforming equations (6) and (7) into frequency domain, we have

$$
\begin{array}{r}
{\left[\begin{array}{cc}
\lambda_{c}+2 \mu & \alpha M \\
\alpha M & M
\end{array}\right]\left[\begin{array}{c}
\nabla^{2} \widetilde{\varphi} \\
\nabla^{2} \widetilde{\chi}
\end{array}\right]=\left[\begin{array}{cc}
-\rho \omega^{2} & -\rho_{f} \omega^{2} \\
-\rho_{f} \omega^{2} & -m \omega^{2}-i \omega b
\end{array}\right]\left[\begin{array}{l}
\widetilde{\varphi} \\
\tilde{\chi}
\end{array}\right],} \\
{\left[\begin{array}{ll}
\mu & 0 \\
0 & \mu
\end{array}\right]\left[\begin{array}{l}
\nabla^{2} \widetilde{\psi} \\
\nabla^{2} \widetilde{\Theta}
\end{array}\right]=\left[\begin{array}{cc}
-\rho \omega^{2} & -\rho_{f} \omega^{2} \\
-\rho_{f} \omega^{2} & -m \omega^{2}-i \omega b
\end{array}\right]\left[\begin{array}{c}
\widetilde{\psi} \\
\widetilde{\Theta}
\end{array}\right] .}
\end{array}
$$

Here, the tilde on variables denotes that they are in the frequency and wavenumber domain.

The above equations can be further manipulated to have the famous Helmholtz equations:

$$
\begin{aligned}
\nabla^{2} \widetilde{\varphi}_{f, s}+\gamma_{f, s}^{2} \widetilde{\varphi}_{f, s} & =0, \\
\left(\nabla^{2}+\gamma_{t}^{2}\right) \widetilde{\psi} & =0,
\end{aligned}
$$

where $\gamma_{f}, \gamma_{s}$, and $\gamma_{t}$ are the wave numbers of two longitudinal and one shear waves, which are written as follows:

$$
\begin{aligned}
\gamma_{f, s}^{2} & =\frac{B \mp \sqrt{B^{2}-4 A C}}{2 A}, \\
\gamma_{t}^{2} & =\frac{C}{D}, \\
A & =(\lambda+2 \mu) M, \\
B & =\left(\lambda_{c}+2 \mu\right)\left(m \omega^{2}+i \omega b\right)+\rho \omega^{2} M-2 \rho_{f} \omega^{2} \alpha M, \\
C & =\rho \omega^{2}\left(h \omega^{2}+\mathrm{i} \omega b\right)-\rho_{\mathrm{f}}^{2} \omega^{4}, \\
D & =\mu\left(m \omega^{2}+\mathrm{i} b \omega\right) .
\end{aligned}
$$

Employing equations (12)-(16), the potentials $\tilde{\varphi}, \tilde{\chi}, \widetilde{\Theta}$, and $\widetilde{\psi}$ can be expressed as follows: 


$$
\begin{aligned}
& \widetilde{\varphi}=\widetilde{\varphi}_{f}+\widetilde{\varphi}_{s}, \\
& \tilde{\chi}=\xi_{1} \widetilde{\varphi}_{f}+\xi_{2} \widetilde{\varphi}_{s}, \\
& \widetilde{\Theta}=\xi_{3} \widetilde{\psi},
\end{aligned}
$$

where

$$
\begin{aligned}
\xi_{1,2} & =\frac{\left(\lambda_{c}+2 \mu\right) \gamma_{f, s}^{2}-\rho \omega^{2}}{\rho_{f} \omega^{2}-\alpha M \gamma_{f, s}^{2}} \\
\xi_{3} & =-\frac{\rho_{f} \omega^{2}}{m \omega^{2}+i b \omega} .
\end{aligned}
$$

The geometry for the ground soils and the tunnel is shown in Figure 1. The soil is a full space with a cavity which has an inner diameter of $a$ while the tunnel is modeled as a thin shell with a radius of the midsurface equal to $a$.

The solution to the potentials in equation (14) can be written as

$$
\begin{aligned}
& \widetilde{\varphi}_{f}=f_{f}(r) \cos n \theta e^{i \xi z}, \\
& \widetilde{\varphi}_{s}=f_{s}(r) \cos n \theta e^{i \xi z}, \\
& \widetilde{\psi}_{r}=g_{r}(r) \sin n \theta e^{i \xi z}, \\
& \widetilde{\psi}_{\theta}=g_{\theta}(r) \cos n \theta e^{i \xi z}, \\
& \widetilde{\psi}_{z}=g_{z}(r) \sin n \theta e^{i \xi z},
\end{aligned}
$$

where $\xi$ is the longitudinal wavenumber and $n$ is a nonnegative integer.

The solution to the functions $f_{f}, f_{s}, g_{r}, g_{\theta}$, and $g_{z}$ are the combinations of modified Bessel as follows:

$$
\begin{aligned}
& f_{f}=A_{f} I_{n}\left(\alpha_{1} r\right)+B_{f} K_{n}\left(\alpha_{1} r\right), \\
& f_{s}=A_{s} I_{n}\left(\alpha_{2} r\right)+B_{s} K_{n}\left(\alpha_{2} r\right), \\
& g_{r}=-g_{\theta}=A_{r} I_{n+1}(\beta r)+B_{r} K_{n+1}(\beta r), \\
& g_{z}=A_{z} I_{n+1}(\beta r)+B_{z} K_{n+1}(\beta r),
\end{aligned}
$$

where $\alpha_{1}^{2}=\xi_{1}^{2}-\gamma_{f}^{2}, \alpha_{2}^{2}=\xi_{2}^{2}-\gamma_{s}^{2}$, and $\beta^{2}=\xi_{3}^{2}-\gamma_{t}^{2} ; I_{\mathrm{n}}$ and $K_{\mathrm{n}}$ are modified Bessel functions ( $n$ represents the order of the functions). The parameters $A_{\mathrm{f}}, B_{\mathrm{f}}, A_{\mathrm{s}}, B_{\mathrm{s}}, A_{\mathrm{r}}, B_{\mathrm{r}}, A_{\mathrm{z}}$, and $B_{\mathrm{z}}$ are unknowns before the application of boundary conditions. The displacements, stresses as well as the pore pressure can be obtained by substituting equation (17) into equations (16), (14), (4), and (5).

For brevity the displacements, stresses as well as pore pressure components are expressed in a concise matrix form as follows:

$$
\begin{aligned}
\tilde{u} & =\left\{\begin{array}{c}
\tilde{u}_{r} \\
\tilde{u}_{\theta} \\
\tilde{u}_{z}
\end{array}\right\} \\
& =[S]\left\{\begin{array}{c}
\tilde{u}_{r n} \\
\tilde{u}_{\theta n} \\
\tilde{u}_{z n}
\end{array}\right\} e^{i \xi z} \\
& =[S][U] C e^{i \xi z}, \\
\tilde{\tau} & =\left\{\begin{array}{c}
\tilde{\tau}_{r r} \\
\tilde{\tau}_{r \theta} \\
\tilde{\tau}_{r z} \\
\tilde{\tau}_{\theta \theta} \\
\tilde{\tau}_{\theta z} \\
\tilde{\tau}_{z z} \\
\tilde{p}
\end{array}\right\}
\end{aligned}
$$

$$
=\left[\begin{array}{ccc}
S & 0 & 0 \\
0 & S & 0 \\
0 & 0 & \cos n \theta
\end{array}\right]\left\{\begin{array}{c}
\tilde{\tau}_{r r n} \\
\tilde{\tau}_{r \theta n} \\
\tilde{\tau}_{r z n} \\
\tilde{\tau}_{\theta \theta n} \\
\tilde{\tau}_{\theta z n} \\
\tilde{\tau}_{z z n} \\
\tilde{p}_{n}
\end{array}\right\} e^{i \xi z}
$$

$$
=\left[\begin{array}{ccc}
S & 0 & 0 \\
0 & S & 0 \\
0 & 0 & \cos n \theta
\end{array}\right][T] C e^{i \xi z},
$$

$$
\text { with }[S]=\left[\begin{array}{ccc}
\cos n \theta & 0 & 0 \\
0 & \sin n \theta & 0 \\
0 & 0 & \cos n \theta
\end{array}\right] \text {, }
$$

where $\mathbf{C}=\left\{A_{f}, B_{f}, A_{s}, B_{s}, A_{r}, B_{r}, A_{z}, B_{z}\right\}^{T}$ is the unknown coefficients vector. The matrices $[\mathbf{U}][\mathbf{T}]$ are given in a previous work done by Yuan et al. [10, 11].

A moving unit point load is expressed in a rectangular coordinate system as follows:

$$
\begin{aligned}
& P_{x}=P_{y}=0, \\
& P_{z}=\frac{\delta(x-c t) \delta(\theta)}{a},
\end{aligned}
$$

where $a$ is the radius of the tunnel; $c$ denotes the velocity of the moving load; and $\delta(x-c t)$ and $\delta(\theta)$ are Dirac delta 


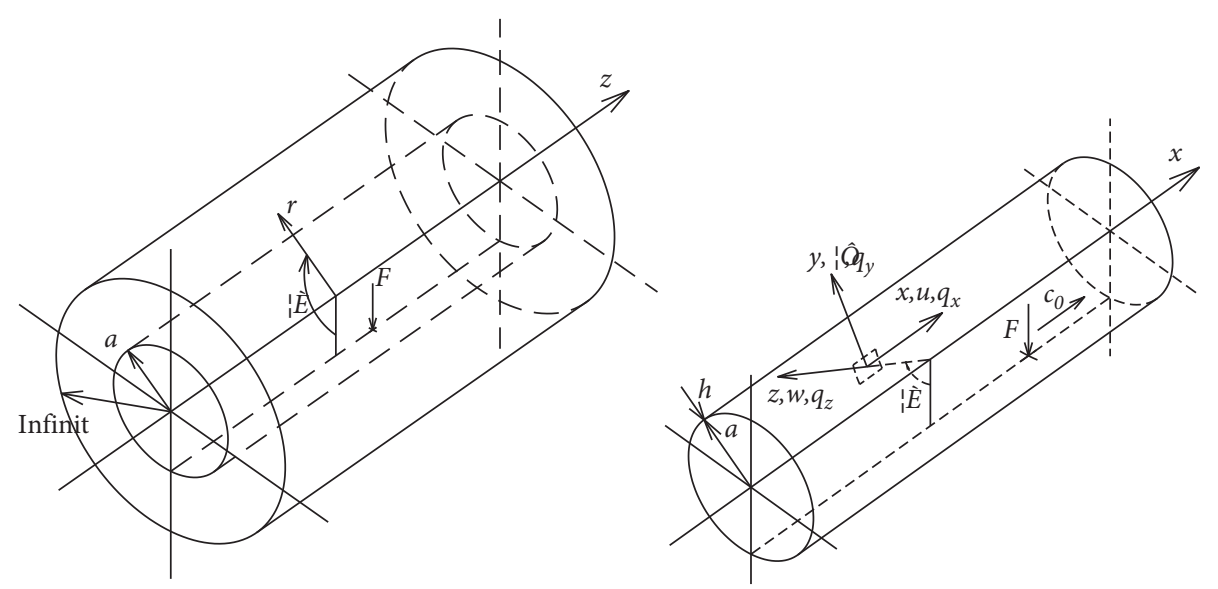

FIgURE 1: The geometry for cavity in soils (a) and the tunnel (b).

functions. $\delta(\theta) / a$ can be expanded into a combination of ring components:

$$
\frac{\delta(\theta)}{a}=\frac{1}{2 \pi a}+\sum_{n=1}^{\infty} \frac{1}{\pi a} \cos n \theta .
$$

The external force can be further expanded into the integration with respect to the wavenumber by the usage of Fourier transformations:

$$
\begin{aligned}
& P_{x}=P_{y}=0, \\
& P_{z}=\frac{1}{2 \pi} \int_{-\infty}^{\infty}\left(\frac{1}{2 \pi a}+\frac{1}{\pi a} \sum_{n=1}^{\infty} \cos n \theta\right) e^{i \xi(x-c t)} \mathrm{d} \xi .
\end{aligned}
$$

Making a comparison with equation (18), the external force for a specific mode number $n$ is

$$
\begin{aligned}
& \widetilde{P}_{x n}=0, \\
& \widetilde{P}_{y n}=0, \\
& \widetilde{P}_{z n}= \begin{cases}\frac{1}{2 \pi a}, & n=0, \\
\frac{1}{\pi a}, & n>0,\end{cases}
\end{aligned}
$$

Therefore, the boundary conditions used to determine $A_{\mathrm{f}}, B_{\mathrm{f}}, A_{\mathrm{s}}, B_{\mathrm{s}}, A_{\mathrm{r}}, B_{\mathrm{r}}, A_{\mathrm{z}}$, and $B_{\mathrm{z}}$ are given as follows:

$$
\left\{\begin{array}{c}
\tilde{\tau}_{r r n} \\
\tilde{\tau}_{r \theta n} \\
\tilde{\tau}_{r z n} \\
\frac{\partial \widetilde{p}_{n}}{\partial r}
\end{array}\right\}=\left\{\begin{array}{c}
\widetilde{P}_{z n} \\
0 \\
0 \\
0
\end{array}\right\} .
$$

For a variable $z$ approaching to infinity, $K_{n}(z)$ tends to zero whereas $I_{n}(z)$ increases to infinity as well. To satisfy the radiation condition at the infinity $r \longrightarrow \infty$, only the modified Bessel functions $K_{n}$ and $K_{n+1}$ can be adopted meaning that

$$
A_{f}=A_{s}=A_{r}=A_{z}=0 \Rightarrow \mathbf{C}=\left\{\begin{array}{llllllll}
0 & B_{f} & 0 & B_{s} & 0 & B_{r} & 0 & B_{z}
\end{array}\right\} .
$$

According to the boundary condition equation (23), the coefficients $B_{f}, B_{s}, B_{r}$, and $B_{z}$ can be determined. Then, by substituting these coefficients into equation (18), the displacement, the stress as well as the pore pressure can be obtained. The response in the time-space domain is written as

$$
\begin{aligned}
\left\{\begin{array}{l}
u_{r} \\
u_{\theta} \\
u_{z}
\end{array}\right\} & =\frac{1}{(2 \pi)^{2}} \int_{-\infty}^{\infty} \int_{-\infty}^{\infty}\left(\left\{\begin{array}{c}
\tilde{u}_{r 0} \\
0 \\
\tilde{u}_{z 0}
\end{array}\right\}+\sum_{n=1}^{\infty}\left\{\begin{array}{c}
\tilde{u}_{r n} \cos n \theta \\
\tilde{u}_{\theta n} \sin n \theta \\
\tilde{u}_{z n} \cos n \theta
\end{array}\right\}\right) e^{i(\omega t+\xi z)} \mathrm{d} \xi \mathrm{d} \omega a \\
& =\frac{1}{(2 \pi)^{2}} \int_{-\infty}^{\infty}\left(\left\{\begin{array}{c}
\tilde{u}_{r 0} \\
0 \\
\tilde{u}_{z 0}
\end{array}\right\}+\sum_{n=1}^{\infty}\left\{\begin{array}{c}
\tilde{u}_{r n} \cos n \theta \\
\tilde{u}_{\theta n} \sin n \theta \\
\widetilde{u}_{z n} \cos n \theta
\end{array}\right\}\right) e^{i \xi(z-c t)} \mathrm{d} \xi .
\end{aligned}
$$


A set of nondimensional variables is introduced with reference length $\left(a_{R}\right)$, shear modulus $\left(\mu_{R}\right)$, and density $\left(\rho_{R}\right)$ as follows:

$$
\begin{aligned}
& r^{*}=\frac{r}{a_{R}}, \\
& z^{*}=\frac{z}{a_{R}}, \\
& \mathbf{u}^{*}=\frac{\mathbf{u}}{a_{R}}, \\
& \lambda^{*}=\frac{\lambda}{\mu_{R}}, \\
& \mu^{*}=\frac{\mu}{\mu_{R}}, \\
& \rho_{f}^{*}=\frac{\rho_{f}}{\rho_{R}}, \\
& \rho^{*}=\frac{\rho}{\rho_{R}}, \\
& c^{*}=\frac{c}{\sqrt{\mu / \rho}}, \\
& m^{*}=\frac{m}{\rho_{R}}, \\
& b^{*}=\frac{a_{R}}{\sqrt{\rho_{R} \mu_{R}}}, \\
& \frac{a_{R}}{\rho_{R}},
\end{aligned}
$$

\section{Numerical Analysis Results and Discussion}

3.1. Numerical Results for a Moving Point Load in a Cavity. The mechanical responses are computed from equation (24). It can be obtained through the summation over the ring components $n$ and transformations over the longitudinal wavenumber $\xi$. An FFT algorithm is employed to compute the integrals with respect to the wavenumber using 4096 integration points and integration step $\Delta \xi=0.04$. The aim of this subsection to present the results for a moving point load in a cavity is twofold. The first is that to compare with the published results in the literature calculated for a ring load. The ring load is different from the point load considered here. However, as the tunnel-soil model is assumed to be a linear system, the principle of superposition is satisfied. Then, to simulate the ring load case available in the published results, the point load can be applied at different positions around the periphery of the cavity surface. Figure 2 compares the results of soil displacement in radial direction and the pore pressure given by Lu [9] with those by the present model. In Lu's work, a moving ring load is applied at the cavity surface (different from the point load case considered here). The model used in the present work can be modified to simulate the model in Lu's work. The whole ring of the tunnel is divided into $360 \Delta \theta$. The displacements are calculated using the present model for each $\theta=n \Delta \theta(n=0,1$, $2, \ldots, 359)$. The total displacements for the present work in Figure 2 are given by the linear combination of the displacement for each $\theta$ as follows:

$$
u_{r}=\sum_{n=0}^{359} u_{r}^{n}(n \Delta \theta) \Delta \theta
$$

The parameters of the soils chosen for the calculation in Figure 2 are the same with those in Lu's work. Figure 2 provides the radial displacement $u_{\mathrm{r}}$ and the pore pressure $p$ versus axial coordinate $z$ for the load velocity equal to half of the shear wave speed of soils $c=0.5 V_{\mathrm{S}}$. The agreement between different models for the mechanical response variables $u_{\mathrm{r}}$ and $p$ confirms the feasibility of the improved tunnel model for poroelastic soils.

As for a moving point load, the critical velocity at which the resonance responses of the system take place is a significant problem for the researchers and industry community. For soft soils with a low shear wave speed, which is usually near the critical velocity of a linear ground system, the velocity of moving sources can easily exceed this critical one leading to the resonance of the system. Regarding the dynamic responses of soils subjected to moving force running on the free surface, the vibrations reach a peak as the load velocity become closer to the Rayleigh wave speed of underlying soils. A moving point load running on a circular tunnel/cavity surface resembles that moving sources on a flat ground surface. It is also expected that there is a critical velocity. This phenomenon is investigated in Figure 3, which gives the displacements varying with $c$ under different permeabilities of the soils. As indicated above in Section 3.1, the velocity and the displacement are provided in a dimensionless way (Table 1). From Figure 3, it is noted that when $c<0.25$, the amplitude of the displacements is rarely influenced by the increase of the load velocity. When $0.25 \leq c \leq 1.0$, the magnitude of the displacements presents a sharp increase as $c$ increases and approaches a maximum value at exactly $c=1.0$. When $c>1.0$, the magnitude of the displacements drops significantly with a further increase in $c$. The influence of permeability parameter $b$ on the displacement as well as the critical velocity is also presented in Figure 3. If the load velocity $c<0.8$, the magnitude of radial displacements decreases as $b$ increases. However, when $0.8 \leq c \leq 1.2$, the amplitude of displacements increases as $b$ increases. When $c>1.2$, again the amplitude of displacements decreases as $b$ increases though the influence of $b$ on the critical velocity is very small.

The spatial distribution of $u_{\mathrm{r}}$ around the periphery of the inner surface of the cavity is shown in Figure 4 for different load moving velocities. As seen in Figure $4,0^{\circ}$ denotes the tunnel invert while $180^{\circ}$ denotes the tunnel apex. Since the model is symmetric, only half of the spatial distribution is provided in Figure 4. At the tunnel invert where the load applied, the peak displacement response appears, and the 

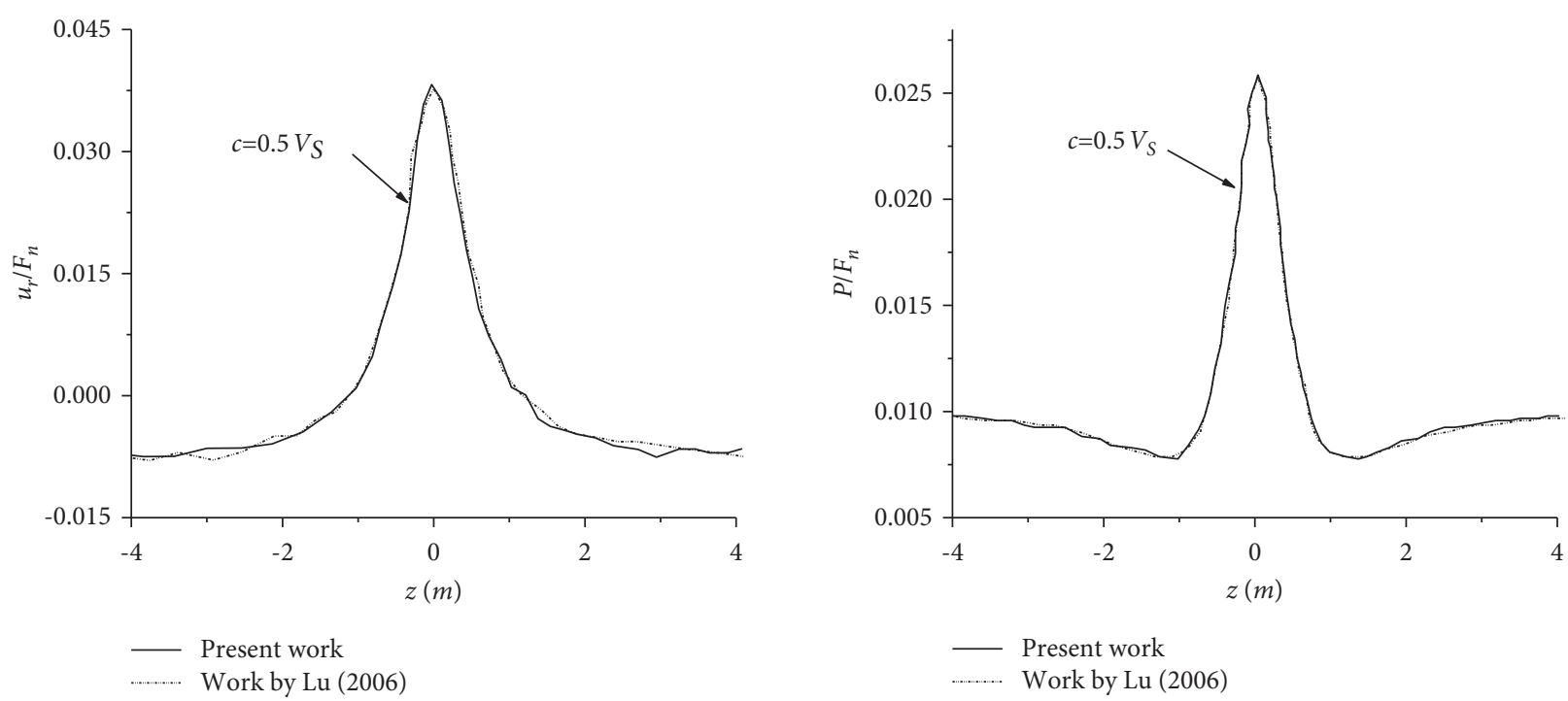

FIGURE 2: Comparison between the numerical result in the work by Lu and Jeng [9] and present work: (a) radial displacement $u_{r} / F_{n}$; $(\mathrm{b})$ pore pressure $p / F_{n}$.

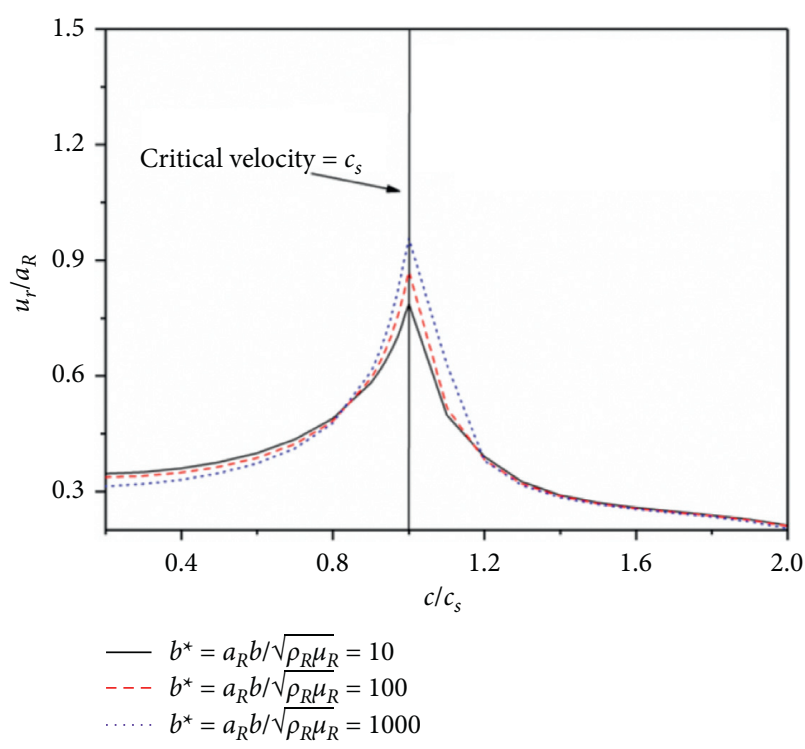

FIgURE 3: $u_{\mathrm{r}}$ varies with $c$ under different $b$.

TABLE 1: Dimensionless parameters of the soils used for Section 3.1.

\begin{tabular}{lc}
\hline Dimensionless coefficients & $\begin{array}{c}\text { Numerical } \\
\text { values }\end{array}$ \\
\hline Lame constants $\lambda^{*}$ & 1 \\
Compressibility of water $M^{*}$ & 1.67 \\
Water density $\rho^{*}$ & 0.53 \\
Hysteretic damping ratio $\beta$ & 0.02 \\
Parameter of soil structure $m^{*}$ & 1.5625 \\
Compressibility of soil grain $\alpha$ & 0.95 \\
Ratio between fluid viscosity and permeability & 10 \\
$b^{*}$ & \\
\hline
\end{tabular}

influence of the load velocity is most significant. With the increase in the observation angle $\theta$, the radial displacement versus the angle $\theta$ fluctuates obviously; however, when the

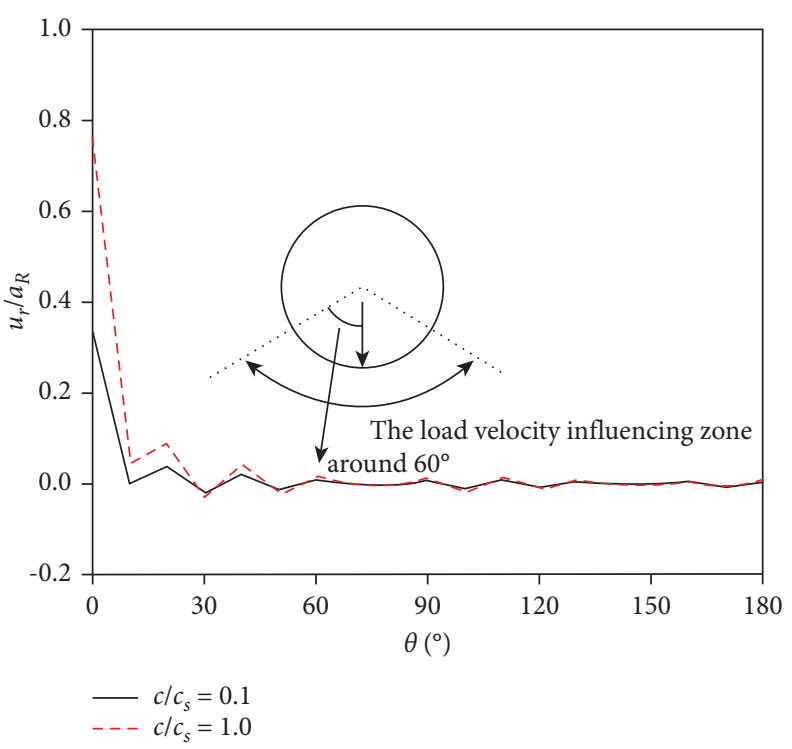

FIGURE 4: The spatial distribution of $u_{r}$ around the periphery of the inner surface of the cavity.

angle is larger than $60^{\circ}$, very small influence can be observed for different moving velocities.

3.2. Numerical Results for a Moving Point Load in a Tunnel. To compare the difference in the mechanical responses for single dry soils and two-phase saturated soils, in Figure 5, a circular tunnel embedded in the dry soils is compared with the results obtained for the two-phase saturated soils. The results for the saturated soils come from a previous work done by Yuan et al. [10]. To guarantee the comparability, the dry soil parameters come from the saturated soil parameters in Yuan's work $[10,11]$. The solid density is $\rho_{\mathrm{s}}=2600 \mathrm{~kg} / \mathrm{m}^{3}$, the porosity $n=0.4$, and the density of pore fluid $\rho_{\mathrm{f}}=0$. This leads to a density of dry soil $\rho_{\mathrm{d}}=\rho_{\mathrm{s}} \times n=1560 \mathrm{~kg} / \mathrm{m}^{3}$. The 

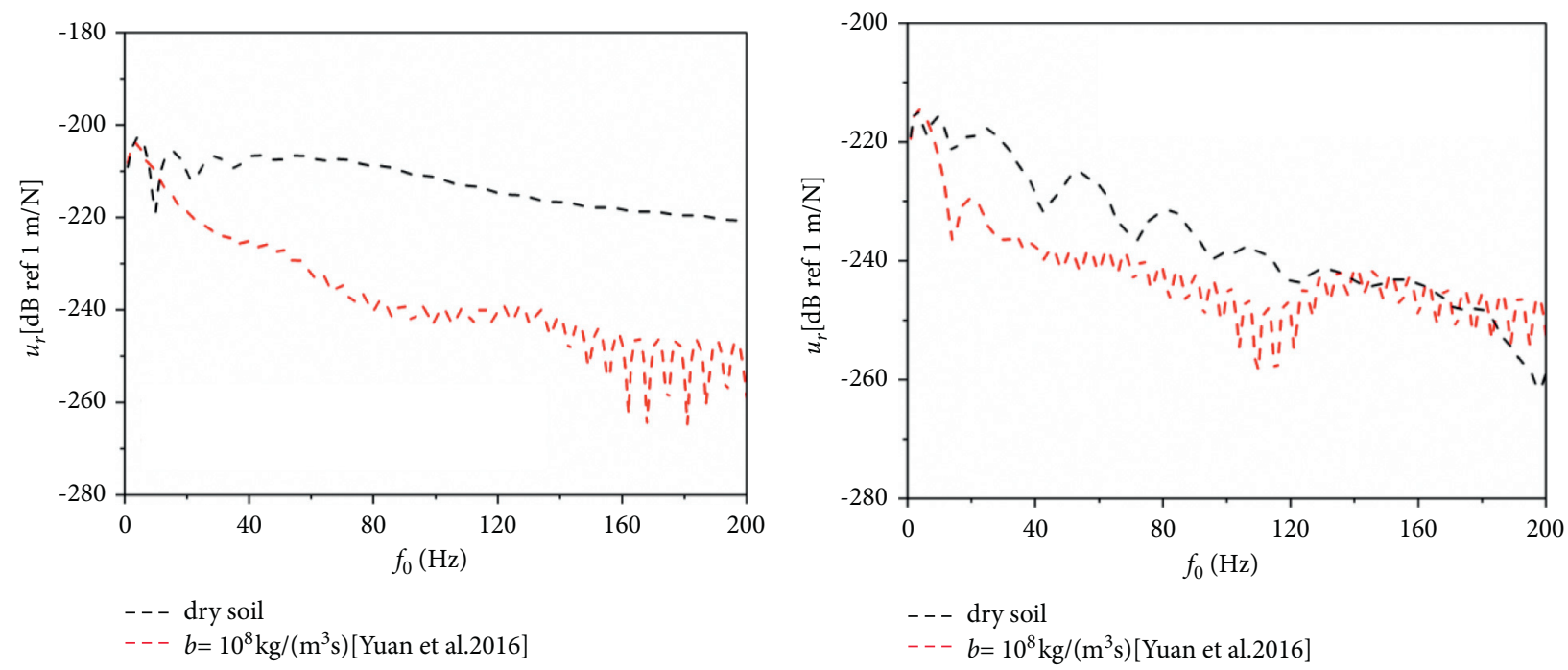

Figure 5: Comparison between single-phase dry soils and two-phase saturated soils: (a) radial displacement $u_{r}$ at point $r=20 \mathrm{~m}, \theta=90^{\circ}$, and $z=0$ and point $\mathrm{r}=20 \mathrm{~m}, \theta=90^{\circ}$, and $z=20 \mathrm{~m}$.

Lame constants $(\lambda$ and $\mu)$ of dry soils are selected as those of the solid skeleton of poroelastic soils. Compared with dry soils, the decreasing trend of the displacement for twophase saturated is more obvious. This is because in addition to the material damping in the soil skeleton, extra viscous damping also exists in two-phase saturated soils because of the motion between the water and solid phase, which leads to more energy loss, in particular in the high-frequency range. On the contrary, as seen in Figure 5, in the lowfrequency range, the differences between the two soil models are not visible. This is because in this frequency range the shear waves dominate the response; however, the addition of the water into the soil skeleton may have small influence on the shear wave properties. Overall, the displacement response for two-phase saturated soils is smaller compared with the response for single-phase dry soils since absolute compression modulus of water is much higher than the solid skeleton.

To further visualize the waves propagated from the tunnel into soils, the magnitude of the soil displacement for a harmonic point source with a frequency $10 \mathrm{~Hz}$ is shown in Figure 6. The displacement magnitude is calculated as $|u|=\sqrt{\left|u_{r}\right|^{2}+\left|u_{\theta}\right|^{2}+\left|u_{z}\right|^{2}}$, where $u_{r}, u_{\theta}$, and $u_{z}$ are the solid displacements in three directions, namely, radial, circumferential, and longitudinal directions. To investigate the effect of presence of water on the mechanical response from a harmonic point excitation, the response magnitude on a transverse-vertical plane for a dry soil model is compared with the result for a saturated soil model (see Figure 6). The contour plots are shown for a concrete tunnel wall buried in a full space and provide a clear inspection into the wave propagation from the tunnel into the soils. As shown in Figure 6, when the frequency is $10 \mathrm{~Hz}$, the energy from the tunnel into surrounding soils is mainly located at the tunnel invert. In general, it can be observed from Figure 6 that the displacements in the case of a tunnel embedded in a saturated soil for $b=1 \times 10^{8} \mathrm{~kg} /\left(\mathrm{m}^{3} \mathrm{~s}\right)$ are smaller than those in the case of a tunnel embedded in a dry soil. This can be explained as the fact that the presence of water increases dynamic stiffness of the tunnel-soil system. More vibration energy is transmitted upwards in the dry soil than that in the saturated soil $\left(b=1 \times 10^{8} \mathrm{~kg} /\left(\mathrm{m}^{3} \mathrm{~s}\right)\right)$. This allows the vibration level in the free field of the saturated soil $\left(b=1 \times 10^{8} \mathrm{~kg} /\right.$ $\left(\mathrm{m}^{3} \mathrm{~s}\right)$ ) above the tunnel smaller than that of the dry soil.

3.3. Numerical Results for a Moving Point Load on a Track. In Figure 7, the influence of load velocity on the rail and track response is investigated using a track-tunnel-soil model. The parameters for the tunnel and soils are the same as those in Section 3.2. The track structure is a floating-slab track consisting of two-layered beams whose parameters are selected from the work done by Hussein et al. [12]. As seen in Figure 7, the resonance peak value occurs at a very high load velocity around $380 \mathrm{~m} / \mathrm{s}$. At the critical velocity, the rail and slab responses for the dry and saturated soils present significant difference while at lower load velocity the presence of water has small influence on the rail and slab responses. It should be noted that at the critical velocity of the tunnel-soil system (shear wave speeds of the soils), the rail and slab responses present a trough value. This is because at this load velocity, the tunnel-soil system is in resonance behavior meaning that its stiffness is low and damping is high, thus leading to a lower track response.

In Figure 8, the influence of load velocity on the soil response is investigated using a fully track-tunnel-soil model. As expected, there are two critical velocities for the coupled system, one is the critical velocity of the tunnel-soil system while the other is the critical velocity of the track itself. In the mechanical response of surrounding soils, both two critical velocities can be observed. Compared with Figure 7 , it is observed that at all the load velocities the influence of presence of water on the soil response is obvious. In a general trend, the dry soil model predicts a higher soil response. 


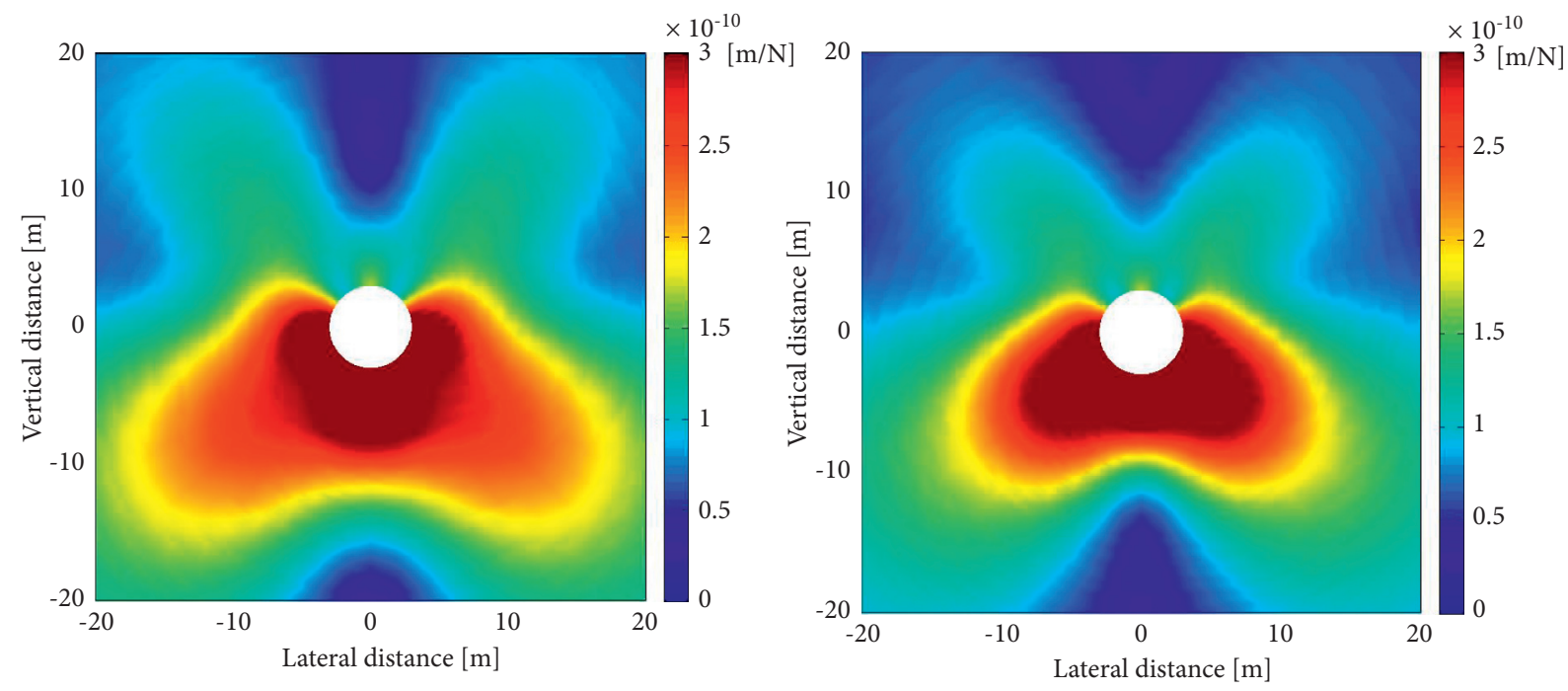

Figure 6: The displacement magnitude around the tunnel for a dry soil model (a) and a saturated soil model (b) with $\left(b=1 \times 10^{8} \mathrm{~kg} / \mathrm{m}^{3} \mathrm{~s}\right)$.

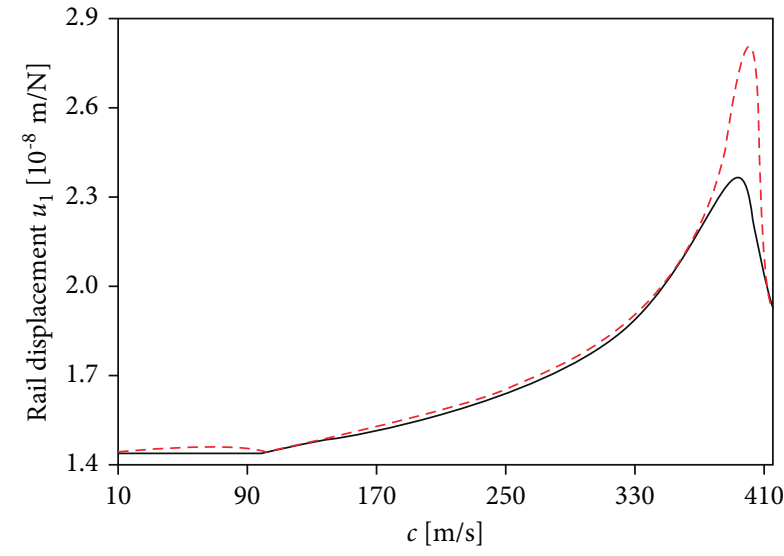

- dry soil

- _ saturated soil with $b=10^{8} \mathrm{~N} \cdot \mathrm{s} / \mathrm{m}^{4}$

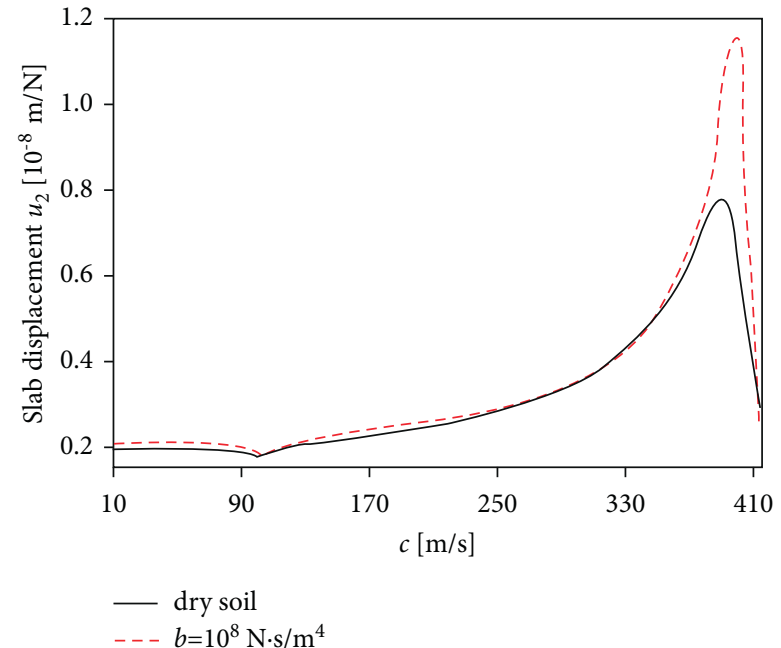

(b) response.

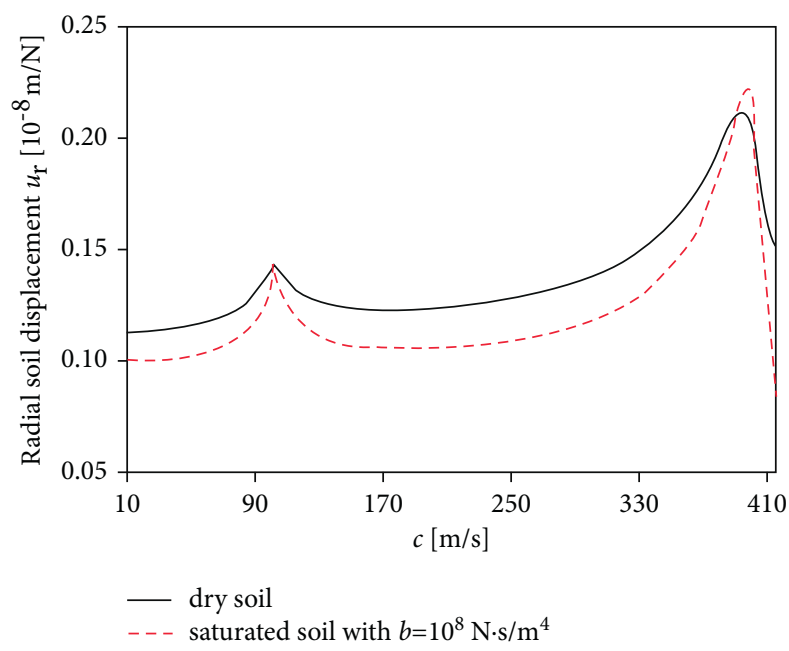

FIgURE 8: The influence of load velocity on the soil response.

\section{Conclusions}

In this work, an improved tunnel-track model in saturated poroelastic soils to a moving point load is proposed. The soils are modeled as two-phase saturated poroelastic materials based on Biot's theory. Using the boundary conditions along the tunnel surface and the full space with a cavity, the mechanical responses such as the displacement, stress, and pore pressure are derived. Numerical results are presented for three cases, namely, mechanical responses for a moving point load in a cavity, in a tunnel and on a track. In particular, the critical velocity for the three cases is analyzed. The main conclusions are summarized as follows:

(1) The present model is suitable for predicting vibrations from underground in the design phase. It can be used to calculate the vibration level around the tunnel; then, with the help of the chain rule for the 
vibration and noise prediction, the preliminary vibration level inside the ground, the foundation, and the nearby buildings can be roughly estimated.

(2) In a general trend, the dry soil model predicts a higher soil response than the two-phase poroelastic soil model; however, the poroelastic soil model provides pore-water pressure response.

(3) The critical velocity for a cavity/tunnel in a full space is around the shear wave speeds of soils while the second one introduced into the track-tunnel-soil system with very high value is around the critical velocity of the track structure itself.

\section{Data Availability}

The data used to support the findings of this study are available from the corresponding author upon request.

\section{Conflicts of Interest}

The authors declare that they have no conflicts of interest.

\section{Acknowledgments}

This work was supported by the National Natural Science Foundation of China (No. 52078462).

\section{References}

[1] W. Gardien and H. G. Stuit, "Modelling of soil vibrations from railway tunnels," Journal of Sound and Vibration, vol. 267, no. 3, pp. 605-619, 2003.

[2] G. Degrande, D. Clouteau, R. Othman et al., "A numerical model for ground-borne vibrations from underground railway traffic based on a periodic finite element- boundary element formulation," Journal of Sound and Vibration, vol. 293, no. 3-5, pp. 645-666, 2006.

[3] D. Clouteau, M. Arnst, T. M. Al-Hussaini, and G. Degrande, "Freefield vibrations due to dynamic loading on a tunnel embedded in a stratified medium," Journal of Sound and Vibration, vol. 283, no. 1-2, pp. 173-199, 2005.

[4] A. V. Metrikine and A. C. W. M. Vrouwenvelder, "Surface ground vibration due to a moving rain in a tunnel: two-dimensional model," Journal of Sound and Vibration, vol. 234, pp. 43-66, 2000.

[5] V. V. Krylov, "Low-frequency ground vibrations from underground trains," Journal of Low Frequency Noise and Vibration, vol. 14, pp. 55-60, 1995.

[6] J. A. Forrest and H. E. M. Hunt, "A three dimensional tunnel model for calculation of train-induced ground vibration," Journal of Sound and Vibration, vol. 294, pp. 678-705, 2006.

[7] F. Guan and I. D. Moore, "Three-dimensional dynamic response of twin cavities due to travelling loads," Journal of Engineering Mechanics, ASCE, vol. 120, pp. 637-651, 1994.

[8] T. Senjuntichai and R. K. N. D. Rajapakse, "Transient response of a circular cavity in a poroelastic medium," International Journal for Numerical and Analytical Methods in Geomechanics, vol. 17, no. 6, pp. 357-383, 1993.

[9] J. F. Lu and D. S. Jeng, "Dynamic response of a circular tunnel embedded in a saturated poroelastic medium due to a moving load," Journal of Vibration and Acoustics, vol. 128, no. 6, pp. 750-756, 2006.
[10] Z. H. Yuan, Y. Q. Cai, and Z. G. Cao, "An analytical model for vibration prediction of a tunnel embedded in a saturated fullspace to a harmonic point load," Soil Dynamics and Earthquake Engineering, vol. 86, pp. 25-40, 2016.

[11] Z. H. Yuan, D. T. Xu, L. Shi et al., "Hybrid analytical-numerical modelling of ground vibrations from moving loads in a tunnel embedded in the saturated soil," European Journal of Environmental and Civil Engineering, pp. 1-29, 2021.

[12] M. F. M. Hussein and H. E. M. Hunt, "Modelling of floatingslab tracks with continuous slabs under oscillating moving loads," Journal of Sound and Vibration, vol. 297, no. 1-2, pp. 37-54, 2006. 